\title{
CHROMOSOME PAIRING IN TRITICUM AESTIVUM cv. CHINESE SPRING
}

\author{
by
}

\author{
GLYN JENKINSI)
}

\author{
Department of Physiology, Carlsberg Laboratory, \\ Gamle Carlsberg Vej 10, DK-2500 Copenhagen Valby \\ 1) Present address: Department of Agricultural Botany, \\ School of Agricultural Sciences, \\ Penglais, Aberystwyth, Dyfed. SY23 3DD. UK
}

Keywords: Synaptonemal complex, interlocking, multivalent, centromere, telomere, prealignment, allopolyploidy, chromatin, zygotene, reconstruction.

Chromosome pairing with the synaptonemal complex in allohexaploid wheat, Triticum aestivum cv. Chinese Spring, has been investigated by serial section series through seventeen pollen mother cell nuclei at the zygotene stage using improved fixation and staining techniques. Homologous chromosomes are not aligned prior to synapsis and exchange of pairing partners and interlocking of chromosomes and bivalents occur at early zygotene. Chromosome knots, the distribution of centromeres and the change in chromatin condensation throughout zygotene are described with particular emphasis on their possible relationship to the resolution of interlockings and multivalents in accordance with the two-phase pairing pattern previously described in Bombyx and wheat.

\section{INTRODUCTION}

Triticum aestivum is an allohexaploid containing three different genomes designated A, B and $\mathrm{D}$. Genetic and cytological investigations have demonstrated that $T$, aestivum behaves as an amphidiploid showing disomic inheritance and 21 bivalents at diakinesis and metaphase I. Hence, crossing over and chiasma formation are restricted to homologous chromosomes within each of the three constituent genomes. This is known to be controlled mainly by a gene or genes on the long arm of chromosome $5 \mathrm{~B}(8,14)$, as its removal or inactivation results in chiasma formation also between homoeologues (15) forming multivalents which persist until metaphase I.

The mechanism by which the gene exerts its 
effect to achieve exclusive bivalent formation has been the subject of much investigation (for review see 16). A widely accepted hypothesis is that proposed by FELDMAN in 1966 (4) which states that in normal dosage, the long arm of chromosome $5 \mathrm{~B}(5 \mathrm{BL})$ suppresses premeiotic association of homoeologues to a greater extent than homologues with the result that the latter are relatively closer together and therefore pair preferentially at zygotene. Premeiotic alignment of homologues is according to this hypothesis a pre-requisite for bivalent formation. Recent observations $(1,2)$ have indeed indicated that in squashed metaphase plates of root-tip cells studied under the light microscope, cytologically marked homologues lie closer together than homoeologues. It has been assumed that this is also the case for the normal morphologically not distinguishable homologues and further that the relative spacial distribution at pre-meiotic interphase is the same as at metaphase in somatic tissue.

The necessity for extrapolation from observations at metaphase is avoided by three dimensional reconstruction of meiocytes from serial electron micrographs, permitting direct observation of the pairing process in cells undisturbed by squashing (for review see 13). In one such investigation (12) it was shown that at zygotene in autotetraploid Bombyx females where crossing over does not occur, multivalents were formed between homologues but were subsequently corrected to bivalents at pachytene. A similar twophase pairing system revealed in triploid Bombyx females (10) led to speculation that multivalents may be formed in wheat and corrected in a similar way before pachytene, thereby accounting for the observation of the exclusive presence of bivalents at metaphase $\mathrm{I}$.

HoBolth (6) fully reconstructed one late zygotene and one early pachytene nucleus of wheat and demonstrated that multivalent formation occurred at zygotene while only bivalents were present at pachytene. He concluded that the transformation of multivalents into bivalents was the mechanism for exclusive bivalent formation in wheat and not a premeiotic alignment of homologues. As HoBOLTH's observations were based on only one fully reconstructed zygotene nucleus, the present study was undertaken in order to further elucidate the pairing behaviour in hexaploid wheat.

A precise understanding of homologous and homoeologous pairing as a basis of meiotic crossing over is of fundamental importance if transfer of genes for plant improvement (e.g. disease resistance, stress tolerance) from closely or distantly related species is to be undertaken with the aid of somatic or sexual hybrids.

\section{MATERIALS AND METHODS}

\subsection{Cultivation of plants}

Seeds of T. aestivum cv. Chinese Spring $(2 n=6 x=42)$ were sown one per pot and grown in a green house at $20^{\circ} \mathrm{C}$ under continuous light. Twenty seeds were planted every week to provide a continuous supply of meiotic material for analysis.

\subsection{Fixation}

\subsubsection{Improvement of fixation}

Fixation damage to at least $80 \%$ of pollen mother cells of wheat at early meiotic prophase was found to occur following conventional glutaraldehyde fixation procedures. In order to allow efficient ultrastructural studies and to ensure that certain meiotic stages were not inadvertantly missed due to differential sensitivity towards fixation, it was necessary to improve the fixation technique. The methods by which improvement was attempted can be grouped in the following way.

(1) Penetration of fixative into anthers: Individual anthers were isolated, cut in half to expose the pollen mother cells and held in the fixative between two thin sheets of agar.

(2) Penetration of fixative into pollen mother cells: As pollen mother cells at zygotene and pachytene are particularly susceptible to fixation damage, it was considered possible that callose, a 1,3- $\beta$-glucan, which surrounds the meiocytes could present a barrier to the penetration of glutaraldehyde. Improvements were attempted, therefore, by (a) increasing the duration and the concentration of the standard fixative, (b) using other highly reactive fixatives such as acrolein and formaldehyde (c) increasing the penetration of glutaraldehyde with dimethyl sulphoxide 
Table I.

Treatments used in the attempt to improve the primary fixation of pollen mother cells. The controls consisted of 20 whole anthers fixed in $4 \%$ glutaraldehyde in $0.1 \mathrm{~m}$-phosphate buffer $\mathrm{pH} 7.4$ for $4 \mathrm{~h}$ at $20^{\circ} \mathrm{C}$. Good fixation was obtained in 14 to 19 of the 80 locules examined per control. The treatments, where quantitative data were not collected because deviation from the control fixation was not obvious, are represented by $=$.

\begin{tabular}{|c|c|c|c|}
\hline Treatment & $\begin{array}{c}4 \% \text { glutaraldehyde } \\
\text { in } 0.1 \mathrm{M} \text {-phosphate } \\
\left(\mathrm{pH} 7.4,20^{\circ} \mathrm{C}\right)\end{array}$ & $\begin{array}{l}\text { Duration of } \\
\text { fixation (h) }\end{array}$ & $\begin{array}{l}\text { Well fixed locules } \\
\text { (per cent of } \\
\text { control) }\end{array}$ \\
\hline Anthers halved & + & 4 & $=$ \\
\hline Anthers halved & + & 24 & 100 \\
\hline $8 \%$ glutaraldehyde & - & 24 & $=$ \\
\hline $10 \%$ acrolein & - & 4 & $=$ \\
\hline $1 \%$ acrolein & + & 4 & $=$ \\
\hline \multicolumn{4}{|l|}{$1.5 \%$ acrolein $+1.5 \%$ paraformaldehyde } \\
\hline$+3 \%$ glurataldehyde & - & 4 & $=$ \\
\hline $2 \%$ DMSO & + & 4 & 24 \\
\hline $4 \%$ DMSO & + & 4 & 0 \\
\hline Pre-treatment with $5 \%$ cellulase $\left(\mathrm{pH} 5.8,37^{\circ} \mathrm{C}, 1 \mathrm{~h}\right)$ & + & 4 & 22 \\
\hline \multicolumn{4}{|l|}{ Pre-treatment with $5 \%$ cellulase $+4 \%$ glutaraldehyde } \\
\hline $\begin{array}{l}\text { Pre-treatment with } 5 \% \text { cellulase }+4 \% \text { glutaraldehyde } \\
\left(\mathrm{pH} 7.4,37^{\circ} \mathrm{C}, 1 \mathrm{~h}\right)\end{array}$ & + & 3 & 31 \\
\hline In vacuo fixation & + & 4 & 249 \\
\hline In vacuo fixation (see text) & + & 8 & 384 \\
\hline \multicolumn{4}{|l|}{ Unbuffered White's tissue culture medium } \\
\hline + 4\% glutaraldehyde & - & 4 & 123 \\
\hline $1,5,10$ and $15 \%$ sucrose & + & 4 & $=$ \\
\hline
\end{tabular}

(DMSO), (d) selectively removing the callose with enzymes and (e) vacuum infiltration during fixation.

(3) Autolysis and osmotic effects: White's tissue culture medium was used instead of phosphate buffer, and sucrose was added to the standard fixative.

The results are presented in Table I. The locules of 20 anthers were examined in transverse thick sections under phase contrast in the light microscope and the percentage of well-fixed locules relative to the control was calculated to allow comparison between treatments of different experiments. The control treatment consisted of fixation in $4 \%$ glutaraldehyde in $0.1 \mathrm{M}$ phosphate buffer ( $\mathrm{pH} \mathrm{7.4)} \mathrm{for} 4 \mathrm{~h}$ at $20^{\circ} \mathrm{C}$. The percentage of well-fixed locules in the controls varied between 18 and 24 . It is clear from Table I that significant improvement occurs only when fixation is performed under vacuum which presumably in some way aids penetration of the fixative by removing air from the anther.

\subsubsection{Fixation in vacuo}

A 4\% solution of glutaraldehyde (purification index, $\mathrm{A}_{235} / \mathrm{A}_{280}$ of 0.1 ) in $0.1 \mathrm{~mm}$-phosphate buffer ( $\mathrm{pH}$ 7.4) containing $1 \mathrm{~mm}-\mathrm{CaCl}_{2}$ and $1 \mathrm{mM}-\mathrm{MgCl}_{2}$ was placed in tubes to which a vacuum line was connected. The tubes were evacuated at room temperature until the dissolved air was expelled and the glutaraldehyde almost reached the boiling point. Tillers at the desired stage of development were excised and transferred to the laboratory with their cut ends immersed in tap water. The leaf sheaths surrounding the spikes were quickly removed and weights were attached to the spikes to ensure complete submersion in the fixative. The spikes were dropped into the tubes containing the fixing solution and the vacuum immediately restored. Fixation in vacuo was allowed to proceed for $8 \mathrm{~h}$ after which the spikes were washed and stored in phosphate buffer overnight at $4{ }^{\circ} \mathrm{C}$. One of the three anthers from each floret was squashed in acetocarmine and viewed under the light microscope 


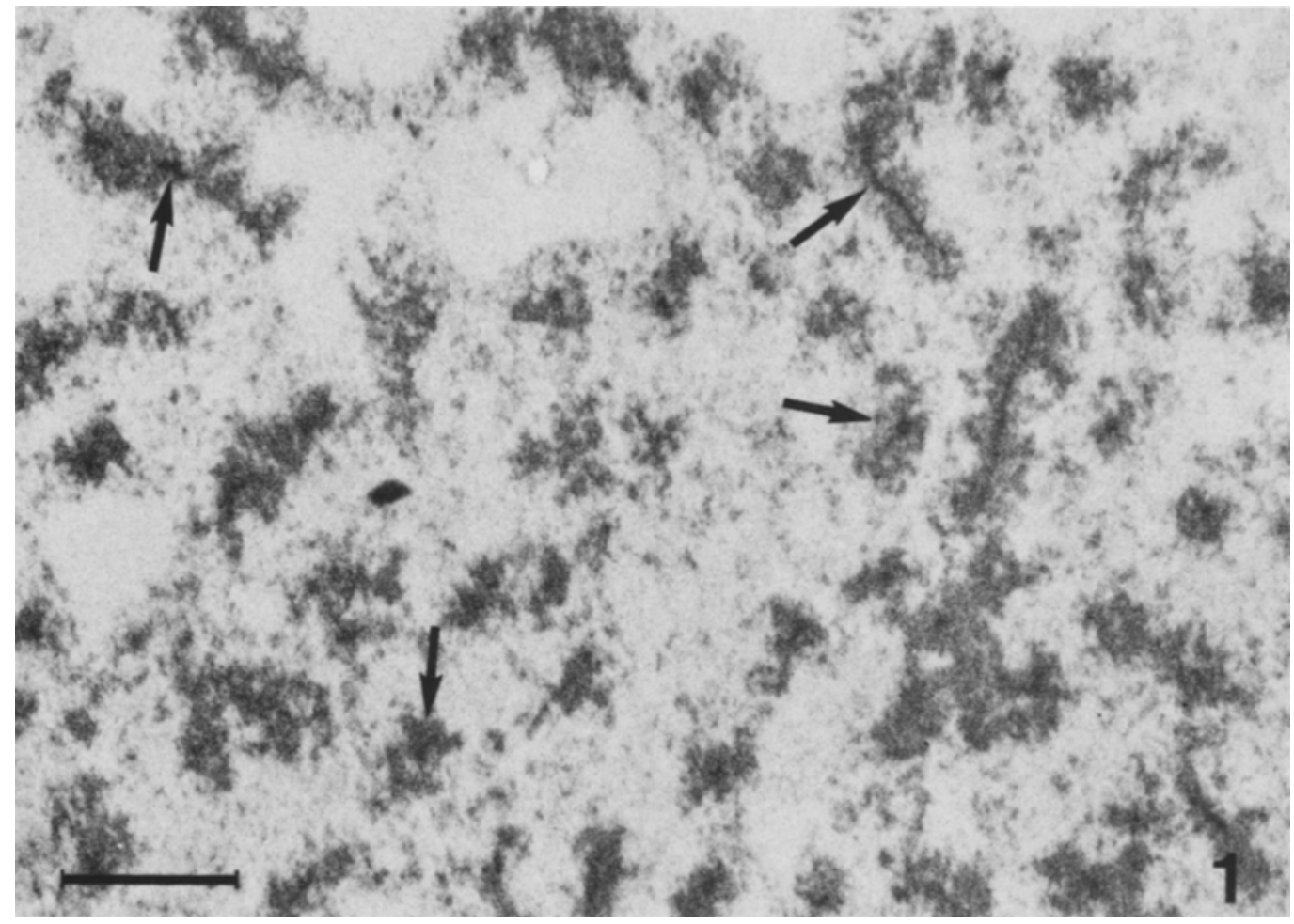

Figure 1. Electron micrograph of a PTA stained pollen mother cell nucleus at early zygotene showing darkly stained lateral components (arrows). (Bar $=1 \mu \mathrm{m}$ ).

to determine the meiotic stage. If suitable, the remaining two were prepared for ultrastructural studies. The fixation of entire spikes eliminates the difficulties in handling isolated anthers and minimises the risk of denaturation during prolonged staging of the anthers prior to fixation. The in vacuo fixation procedure regularly ensured that at least $70 \%$ of the wheat pollen mother cells selected at zygotene and pachytene were useful for electron microscopic investigation and may also improve fixation of pollen mother cells of other cereals and grasses.

Following glutaraldehyde fixation the selected anthers were subjected to one of two treatments. Some were post-fixed in a $2 \%$ solution of osmium tetroxide in $0.1 \mathrm{M}$-phosphate buffer $(\mathrm{pH}$ 7.4) for $2 \mathrm{~h}$ at room temperature followed by two rinses in distilled water and dehydration in a graded ethanol series. Others were dehydrated immediately and stained in a $1 \%$ phosphotungstic acid (PTA) solution in ethanol (17) at $4{ }^{\circ} \mathrm{C}$ overnight followed by two washes in ethanol.
PTA was found to stain both single and paired lateral components of the synaptonemal complex more intensely than the chromatin in zygotene pollen mother cells (Figure 1) and hence facilitated the three dimensional reconstruction of the chromosomes. All anthers were then washed in propylene oxide and infiltrated with Spurr's low viscosity resin (18) which was polymerised overnight at $70{ }^{\circ} \mathrm{C}$. Thick survey sections of the embedded anthers were examined under phase contrast in the light microscope for a preliminary identification of meiotic stages. Thin survey sections were taken from potentially useful anthers for precise stage determination before selecting those for thin serial sectioning. All sections were cut on a Reichert OM U3 microtome equipped with a diamond knife. Serial sections of nominally $100 \mathrm{~nm}$ thickness of the osmicated material were double-stained in $5 \%$ uranyl acetate for $30 \mathrm{~min}$ at $60^{\circ} \mathrm{C}$ and in lead citrate for $30 \mathrm{~min}$ at room temperature in an LKB Ultrostainer 2168, System Carlsberg. The 
PTA stained sections required no further treatment. The serial sections were examined in a Siemens Elmiskop 102 electron microscope at $80 \mathrm{kV}$ and micrographs of selected nuclei were taken at a primary magnification of 3000 times and printed to a final magnification of between 14,700 and 21,000 times. Three dimensional reconstructions of the lateral components, together with the length measurements were performed as described by RASMUSSEN and HOLM (11). In the present study, 17 nuclei were serially sectioned and photographed (119 to 194 sections per nucleus) and the chromosomes of one early zygotene nucleus were fully traced onto transparent plastic (Figure 13).

\section{RESULTS}

\subsection{Substaging of zygotene}

Zygotene is defined as the interval of meiosis during which chromosome pairing and synaptonemal complex formation occur. Hence, the degree of pairing can be used as a criterion for substaging zygotene. In 15 complete series through zygotene nuclei, the progress of chromosome pairing was estimated using both the frequency of paired centromeres and of chromatin blocks associated with a synaptonemal complex as observed on the median section of each nucleus. The former was not considered to be an accurate enough estimate alone as centromeres represent only a small fraction of the total length of chromosomes and, as shown in section 3.2., centromeres are probably one of the last segments of the chromosomes to pair. A measurement of the degree of chromosome synapsis based on centromeres will, therefore, almost certainly be an underestimate. This notion is supported by the data in Table II which show that in medial sections, the percentage of centromere pairing was without exception less than the percentage of chromatin blocks paired with a synaptonemal complex. More importantly, however, there is generally a very good correlation between the two estimates in each nucleus i.e. when the number of paired centromeres is low, the number of chromatin blocks associated with the synaptonemal complex is also low, and vice versa. This agreement permitted the use of the two parameters to arrange the 15 nuclei in an approx-
Table II.

Intranuclear features of 15 zygotene pollen mother cells ranked (youngest to oldest) according to degree of pairing. Nuclei 1 to 5, 7 and 8, 10 and 14, and 11,12 and 15 belong, respectively, to the same anthers.

\begin{tabular}{rccc}
\hline & $\begin{array}{c}\text { Paired } \\
\text { centromeres } \\
\text { Nucleus }\end{array}$ & $\begin{array}{c}\text { Paired chromatin } \\
\text { (plocks paired with } \\
\text { a synaptonemal } \\
\text { complex (per cent) }\end{array}$ & $\begin{array}{c}\text { Number of } \\
\text { chromosome } \\
\text { knots }\end{array}$ \\
\hline 1 & 0 & 33 & 2 \\
2 & 0 & 39 & 3 \\
3 & 5 & 36 & 3 \\
4 & 5 & 40 & 3 \\
5 & 5 & 46 & 6 \\
6 & 0 & 48 & 6 \\
7 & 5 & 59 & 2 \\
8 & 5 & 63 & 1 \\
9 & 38 & 64 & 1 \\
10 & 43 & 76 & 0 \\
11 & 52 & 83 & 0 \\
12 & 67 & 86 & 0 \\
13 & 67 & 87 & 0 \\
14 & 76 & 90 & 0 \\
15 & 81 & 93 & 0 \\
\hline
\end{tabular}

imate temporal sequence as shown in Table II. It will be noticed that nuclei derived from the same anther do not in all cases rank in positions next to one another. This variation in degree of pairing is probably attributable to some asynchrony in the pairing process between cells within the same anther.

\subsection{Centromere distribution and pairing}

Centromeres are morphologically distinct structures throughout zygotene and appear as either paired or unpaired regions less electron dense than the chromatin (Figure 2). The spatial distribution and pairing characteristics of the centromeres of the 15 zygotene nuclei were analysed (Table III) typical cells being represented by the diagrams in Figure 3. Firstly, it will be seen that in most nuclei the centromeres exhibit a distinct polarisation, the direction of which is apparently unrelated to the position of the pollen mother cell in the anther. It is considered likely that the localisation of centromeres is the result of telomeric polarisation which is evident from the reconstruction of synapsing bivalents at early zygotene (section 3.4). It will also be noticed 



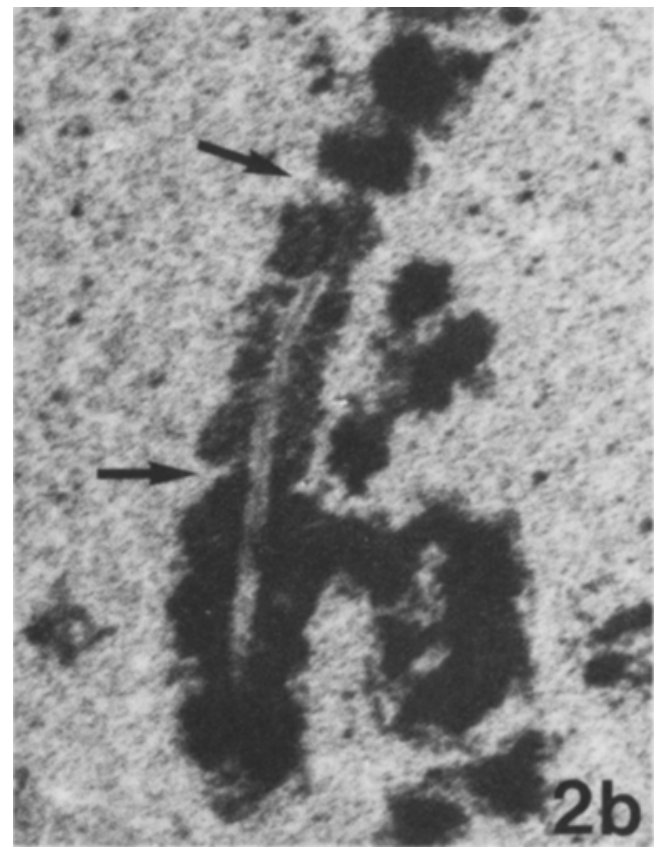

Figure 2. a. Unpaired centromere in longitudinal section at early zygotene (glutaraldehyde-osmium tetroxide fixation). b. Two longitudinally sectioned centromeres paired with a synaptonemal complex at midzygotene (glutaraldehyde -osmium tetroxide fixation). c. Paired centromeres with synaptonemal complex in transverse section at late zygotene (glutaraldehyde fixation, PTA stained). Extent of the centromeres are indicated by arrows. $(\mathrm{Bar}=1 \mu \mathrm{m})$. from Figure 3 and Table III that centromeres aggregate, as has been seen previously in wheat at pre-meiotic interphase (3). It is not possible to assess whether or not two centromeres are homologous. However, clusters of 3 and 4 cen- tromeres cannot all be homologous and unequivocally show that non-homologous and/or homoeologous centromeres also associate during zygotene. The reduction in number of aggregates with two centromeres towards late 
Table III.

Centromere distribution and pairing characteristics in 15 zygotene nuclei.

\begin{tabular}{|c|c|c|c|c|c|c|}
\hline \multirow[t]{2}{*}{ Nucleus } & \multicolumn{2}{|c|}{$\begin{array}{l}\text { Number of } \\
\text { centromeres }\end{array}$} & \multicolumn{3}{|c|}{$\begin{array}{c}\text { Number of aggregates } \\
\text { with } 2,3 \text { or } 4 \\
\text { centromeres } \\
\end{array}$} & \multirow[t]{2}{*}{$\begin{array}{c}\text { Distinct } \\
\text { polarisation }\end{array}$} \\
\hline & Paired & Unpaired & 2 & 3 & 4 & \\
\hline 1 & 0 & 42 & 6 & 1 & 0 & - \\
\hline 2 & 0 & 42 & 3 & 4 & 0 & + \\
\hline 3 & 2 & 40 & 4 & 3 & 0 & + \\
\hline 4 & 2 & 40 & 2 & 1 & 0 & + \\
\hline 5 & 2 & 40 & 3 & 2 & 2 & + \\
\hline 6 & 0 & 42 & 5 & 1 & 0 & + \\
\hline 7 & 2 & 40 & 3 & 0 & 0 & + \\
\hline 8 & 2 & 40 & 3 & 1 & 0 & + \\
\hline 9 & 16 & 26 & 5 & 1 & 0 & + \\
\hline 10 & 18 & 24 & 1 & 0 & 0 & - \\
\hline 11 & 22 & 20 & 0 & 1 & 1 & + \\
\hline 12 & 28 & 14 & 0 & 1 & 1 & + \\
\hline 13 & 28 & 14 & 0 & 0 & 0 & - \\
\hline 14 & 32 & 10 & 0 & 1 & 0 & - \\
\hline 15 & 34 & 8 & 1 & 0 & 0 & + \\
\hline
\end{tabular}

zygotene is attributable to their pairing with a synaptonemal complex.

Centromeres paired to non-centromeric chromatin with a stretch of synaptonemal complex, a pairing which is obviously non-homologous, was observed in few cases.

\subsection{Chromatin morphology}

Electron micrographs of the median section from 5 nuclei are arranged in Figures 5-9 in a temporal sequence based on the extent of chromosome pairing and show a progressive condensation of the chromatin during the first part of zygotene followed by a decondensation towards the end of zygotene. The sequence is not absolute as some of the later nuclei of the same anther, e.g. 10 and 14, display similar degrees of condensation but vary with respect to the degree of chromosome synapsis. Assuming the latter to be correctly estimated and to be a reasonable measure of stage of development, there is, therefore, in some cases lack of correlation between chromatin condensation and progress of chromosome pairing. However, it has been shown in previous reports $(3,6)$ that a zygotene substage with highly condensed chromatin occurs well before chromosome pairing is complete and that this is followed by a stage with decondensed chro- matin at late zygotene and early pachytene (6).

A prominent feature of the early zygotene nuclei is knots involving several, and in some cases many, paired as well as unpaired chromosomes. The knots either appear in an open configuration with loss of lateral component continuity in the vicinity of the knot (Figure 10) or possess a central core which disrupts the continuity of the chromosomes which enter it (Figure 11). The central core stains heavily with PTA and lightly with osmium tetroxide. The number of knots in each of the 15 zygotene nuclei is given in Table II. It can be seen that their number increases during the first part of zygotene and decreases to zero as zygotene proceeds. Although in mid zygotene nuclei knots are few or no longer present, close chromatin contact at the periphery of bivalents is still evident which gives the chromatin a reticulate appearance as seen in Figure 7. In further progressed nuclei ( 11 and 12 ) the highly condensed chromatin of bivalents appears linked by chromatin "bridges". In many cases these apparent "bridges" may simply be due to the intertwining of unpaired chromosome segments and univalents (Figure 12) or reflect pairing partner exchange. By late zygotene (Figure 9) bivalents appear as individual, distinct units with few connections to other bivalents along their lengths. 

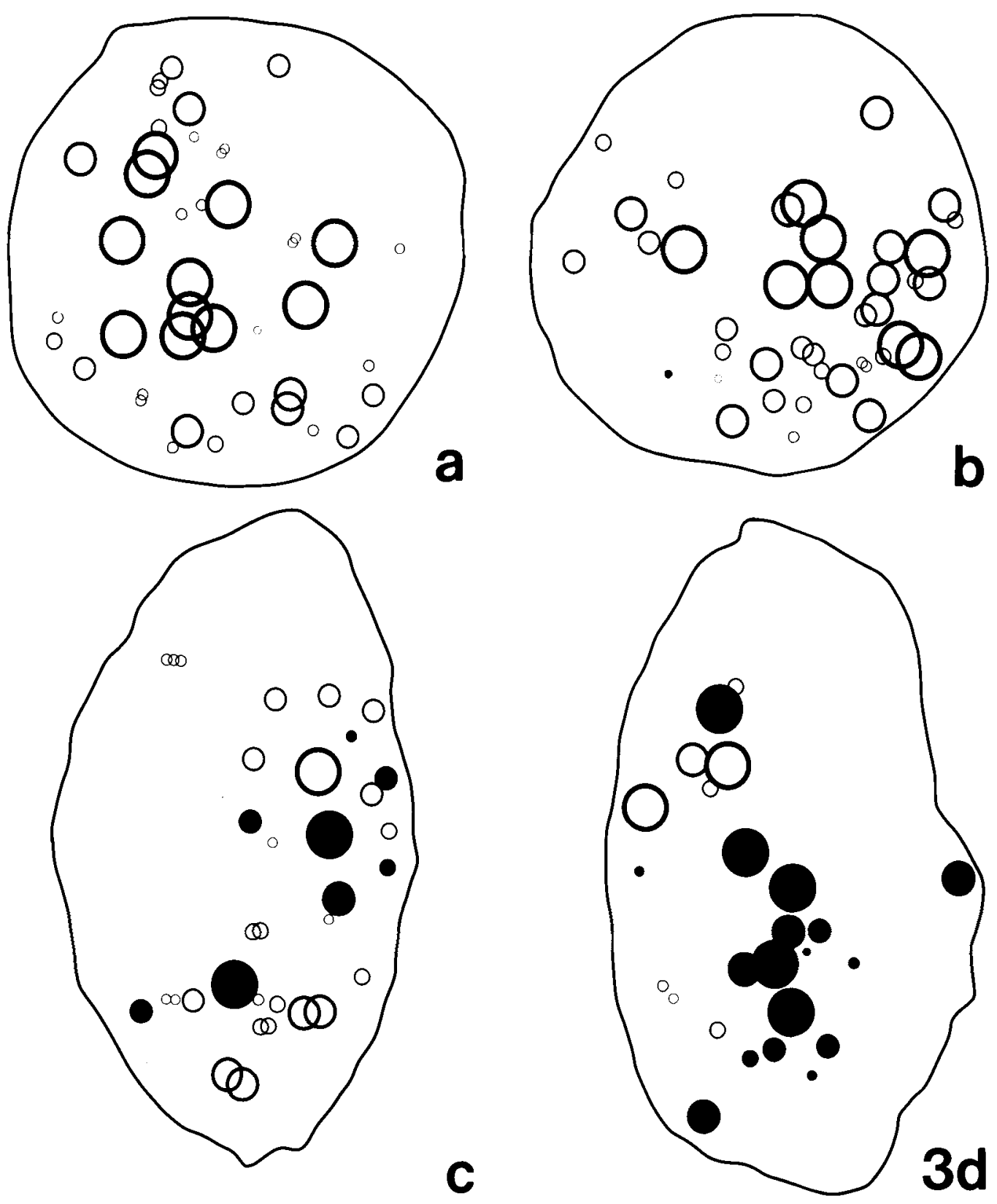

Figure 3. Centromere distribution in nuclei 1 (a), 7 (b), 9 (c) and 15 (d) of Table II. Unpaired centromeres are represented by open circies and paired ones by shaded circles. The size of the circles indicates its location in the nucleus, large circles denoting a position towards the viewer and small ones towards the opposite surface of the nucleus. The tapetum runs tangentially to the left of each nucleus. Note the distinct polarisation of centromeres in Figures b, $c$ and d. (Magnification approximately 3,600 times).

Thus, it seems that during zygotene there is a progressive diminution of close chromosome contact other than that of homologues within bivalents. The significance of this observation with regard to the resolution of certain chromosome associations is discussed later. 
Figure 4. a, b. Consecutive sections through a centromere (ce) paired by a synaptonemal complex with a non-centromeric chromosome region c. Mispaired centromere with polycomplex (arrow). $(\mathrm{Bar}=1 \mu \mathrm{m})$.

\subsection{Chromosome distribution at early zygotene}

As PTA was found to selectively stain the lateral components of the chromosomes at early zygotene (Figure 1), it was possible to completely trace onto plastic sheets the entire chromosome complement of one nucleus at a stage comparable to that of nuclei 1 to 5 of Table II. Figure 13 shows the original tracings stacked in such a way as to give a good visual impression of the general distribution of chromosomes through the early zygotene nucleus. It is obvious from Figure 13 that there is a distinct polarisation of paired telomeres. The restriction of telomeres to a limited area on the nuclear envelope during leptotene and zygotene has been seen in wheat (6) and in other plants, e.g. maize (5) and lily (7), although a distinct chromosome bouquet is not evident as the chromosomes span the nuclear diameter several times. Secondly, synaptonemal complexes are preferentially located in the part of the nucleus which contains the attachment sites of the telomeres. This pattern is not confined to the fully traced nucleus as 7 out of 9 of the early zygotene nuclei show some degree of polarisation of paired chromatin blocks on the median section (Table II). This shows that chromosome pairing and synaptonemal complex formation begin preferentially at the telomeres.

Another important observation in the nucleus of Figure 13 is the complex arrangement of unpaired lateral components which appear to be randomly distributed with respect to one another with no apparent alignment prior to synapsis.

\subsection{Chromosome pairing}

Although the chromosome knots described above effectively prevented a complete reconstruction of the early zygotene nucleus, it was possible to reconstruct some bivalents fully or partially, a representative selection being shown in Figures 14-16. It will be noted that the telomeres of all three bivalents are paired and are
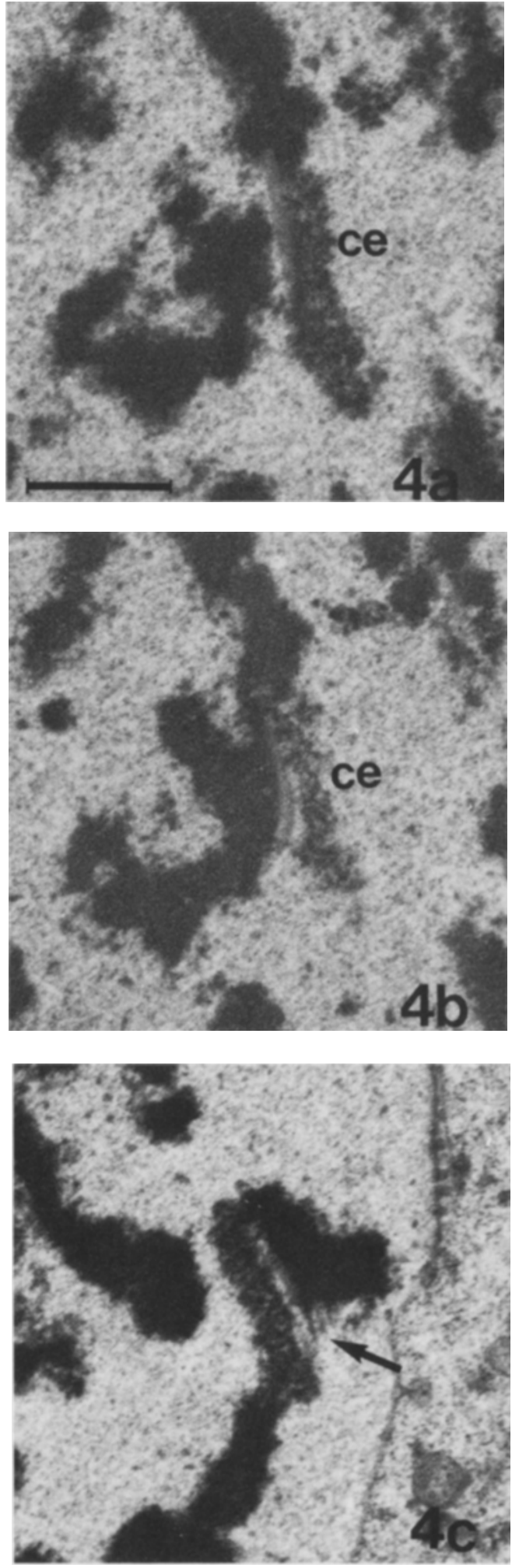




Figure 5. Median section through a glutaraldehyde-osmium fixed early zygotene nucleus (nucleus 1, Table II). (Bar $=2 \mu \mathrm{m}$ ). 




Figure 6. Median section through a PTA stained early zygotene nucleus (nucleus 2, Table II). $(\mathrm{Bar}=2 \mu \mathrm{m})$. 


\section{G. JENKINS: Zygotene in allohexaploid wheat}

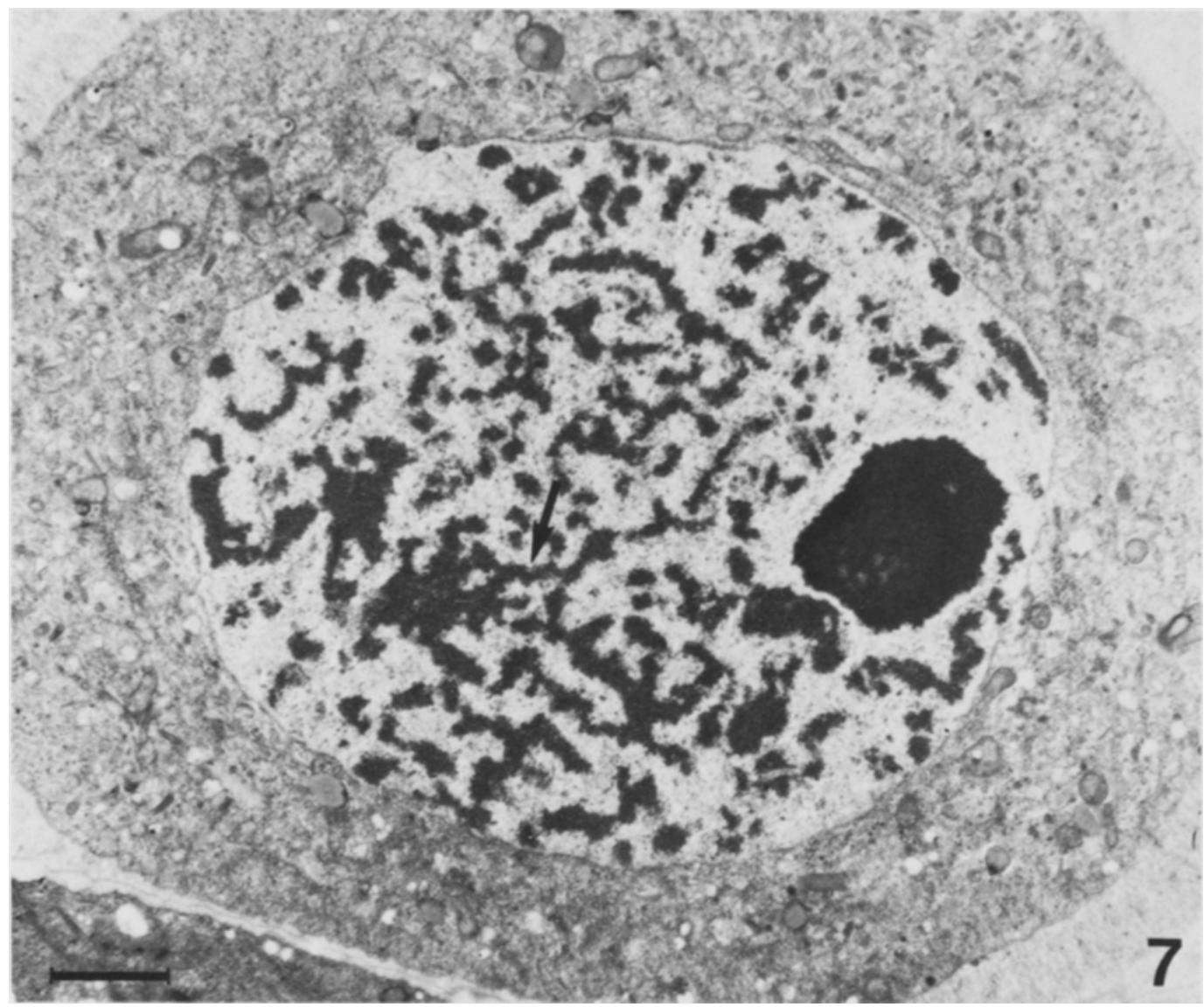

Figure 7. Median section through a glutaraldehyde-osmium fixed mid- zygotene nucleus (nucleus 8 , Table II). Note the reticulate appearance of the chromatin (arrow). $(\mathrm{Bar}=2 \mu \mathrm{m})$. 


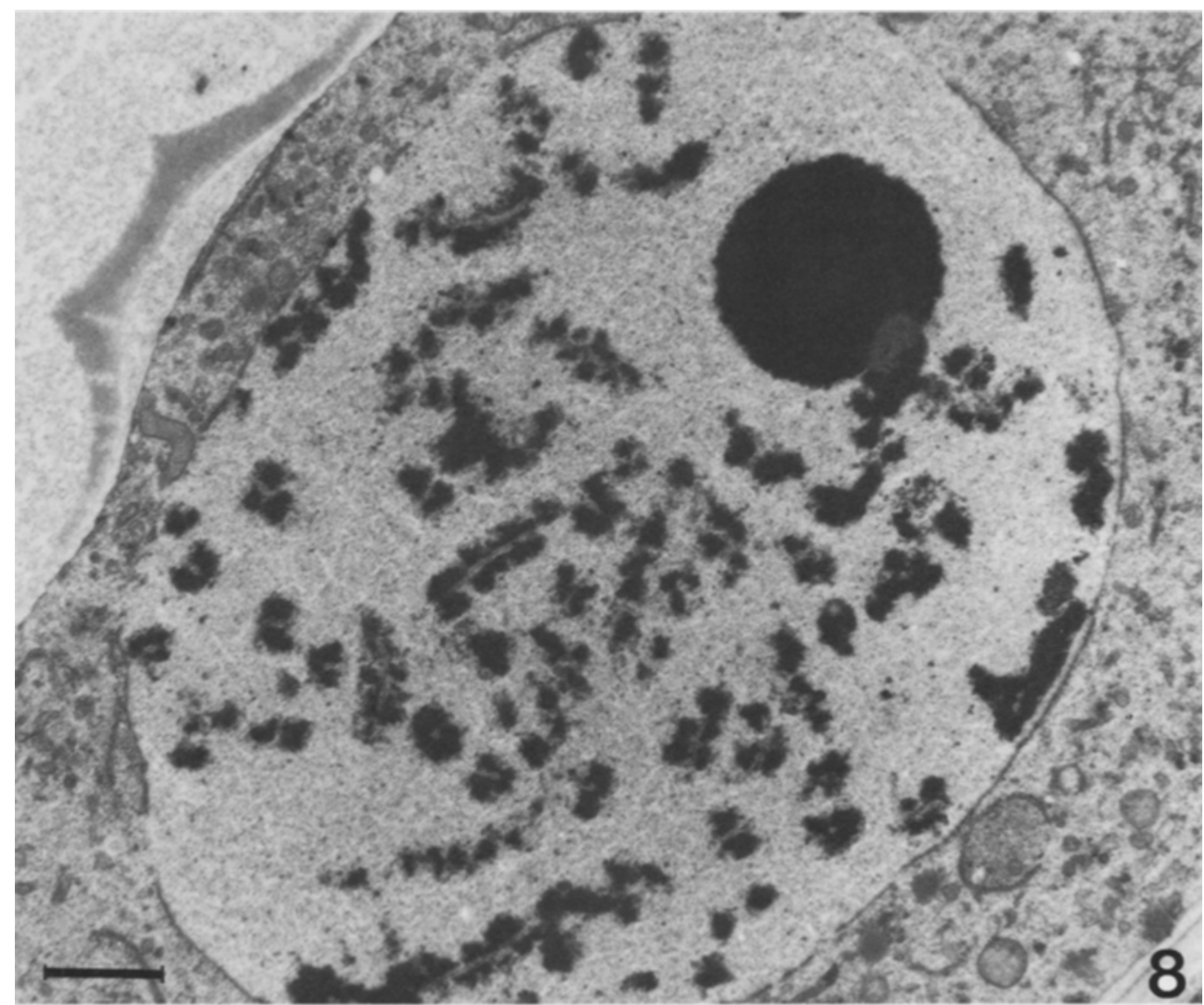

Figure 8. Median section through a glutaraldehyde-osmium fixed mid- zygotene nucleus (nucleus 11, Table II). Note the highly condensed chromatin. $(\mathrm{Bar}=2 \mu \mathrm{m})$. 
G. JENKINS: Zygotene in allohexaploid wheat

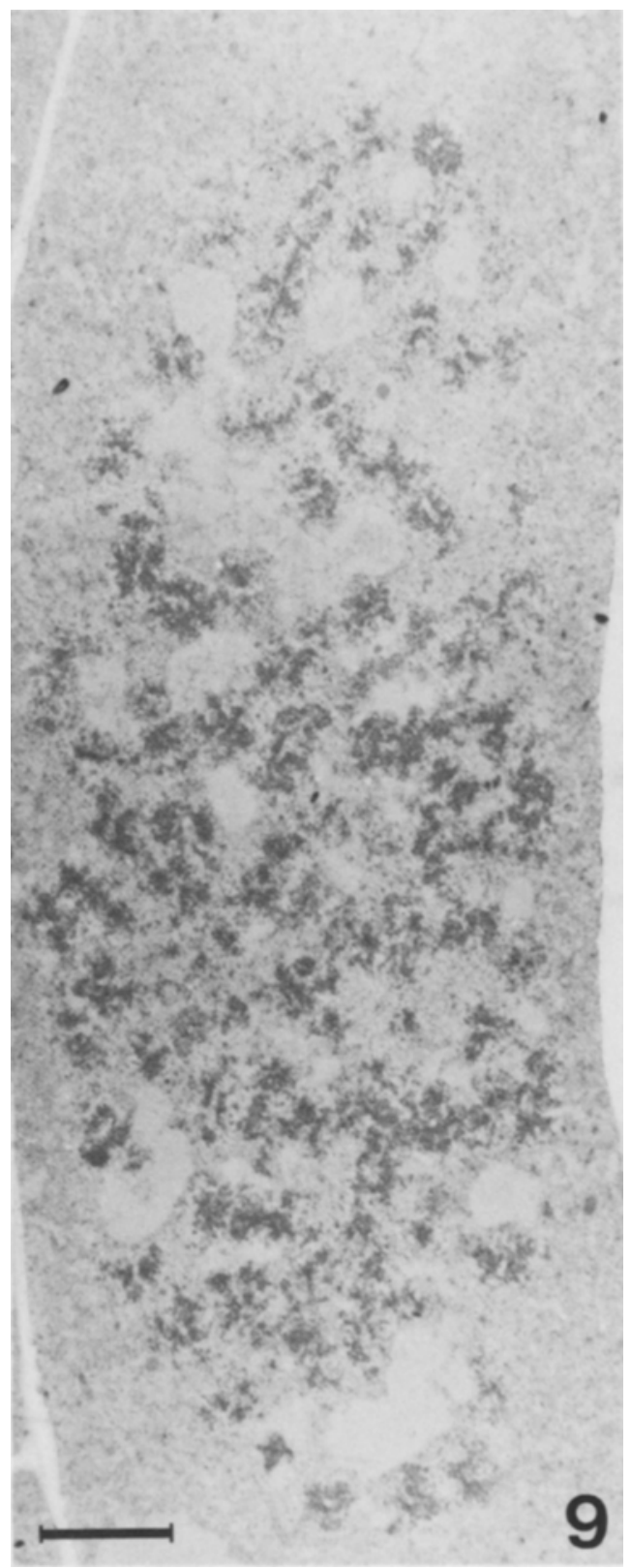

Figure 9. Median section through a PTA stained late zygotene nucleus (nucleus 15 , Table II). (Bar $=2 \mu \mathrm{m})$. 



Figure 10. a-d. Consecutive section series through a PTA stained open knot. e. Diagram of chromosome segments involving unpaired lateral components (single lines) and synaptonemal complexes (parallel lines with cross hatching) entering a 'knot' (shaded region). Note the loss of lateral component continuity inside the knot (arrows). $(\mathrm{Bar}=1 \mu \mathrm{m})$. 



Figure 11. Sections through PTA stained (a) and glutaraldehyde-osmium fixed (b) knots possessing a prominen central core (arrows). $(\mathrm{Bar}=1 \mu \mathrm{m})$.

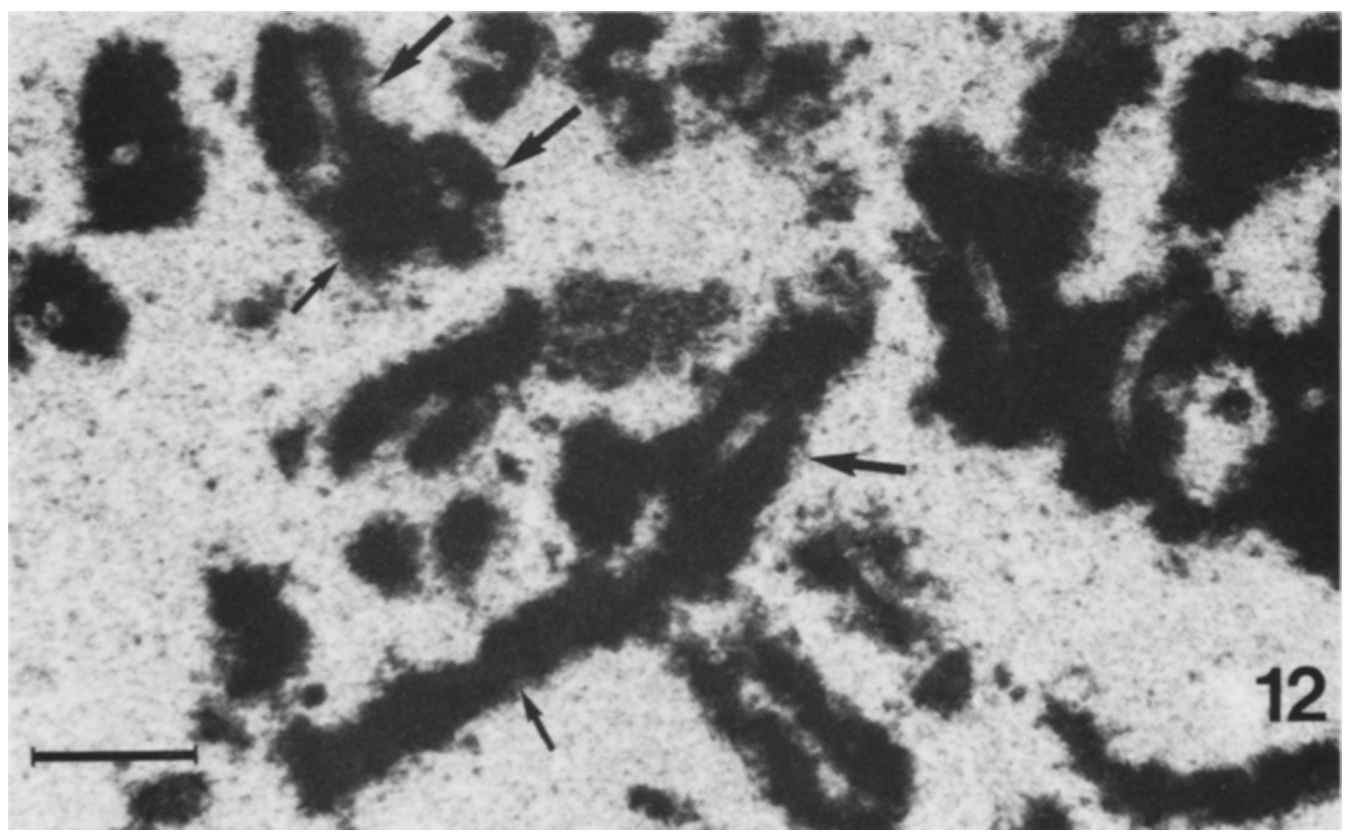

Figure 12. Electron micrograph showing intertwining of unpaired chromosome segments (small arrows) in direc contact with bivalents (large arrows) in a mid-zygotene nucleus with highly condensed chromatin. $(\mathrm{Bar}=1 \mu \mathrm{m})$. 
attached to the nuclear envelope in the same vicinity. The significance of the localisation of paired telomeres is not known but the data are consistent with the observation in other organisms that attachment to the nuclear envelope somehow facilitates the recognition of homologues and initiation of synaptonemal complex formation (13).

Pairing of lateral components into the synaptonemal complex can also occur interstitially in wheat as in Lilium (7) and Zea (5), often with distinct pairing forks of the lateral components (Figure 17). It is not possible to know from the available data if interstitial synapsis precedes, follows or is simultaneous with telomeric pairing as no bivalents with either telomeric or interstitial pairing alone were reconstructed.

As illustrated in Figure 14, long unpaired lateral components of partially paired bivalents undergo complex twisting and foldback through large portions of the nucleus with homologous regions such as the centromeres being widely separated.

The primary tracing of the chromosome complement (Figure 13) revealed potential chromosome and bivalent interlockings in a number of regions but as the entire chromosome complement was not completely reconstructed unambiguous identification of interlockings was not possible. An example of a possible multiple interlocking is depicted in Figure 18 where a number of chromosomes and bivalents are located between the unpaired lateral components in the middle region of the bivalent shown in Figure 14.

An additional feature of the nucleus is a multivalent. It was possible to unequivocally determine that at least 6 chromosomes are involved in a complex pairing configuration (Figure 19). This is consistent with the observations of HoBOLTH (6) who saw 4 quadrivalents, 1 hexavalent and 1 pentavalent in a completely reconstructed late zygotene nucleus. The multivalent consists of five chromosomes each involved in extensive synaptonemal complex formation ending at membrane-bound telomeres, and a small stretch of synaptonemal complex with an additional chromosome (shown as a segment only). It can be seen that synaptonemal complex formation is initiated at many sites as in the three reconstructed bivalents and that shifts of pairing part- ners occur in interstitial regions. This complex pairing configuration differs from those reported by HoвоLтн (6) in that only one exchange of pairing partners occurs between any two chromosomes and not an even number as seen at late zygotene. This means that, although it is not possible to identify the chromosomes even by relative length, much of the synaptonemal complex formed is between chromosomes that are not homologues.

Non-homologous pairing, or less likely heterozygosity for an inversion, was identified in one instance in the partially reconstructed early zygotene nucleus. The short segment, denoted by an arrow in Figure 16 combines two lateral components of opposite polarity into a synaptonemal complex.

\section{DISCUSSION}

The present work has shown that long unpaired stretches of chromosomes are randomly distributed and are involved in complex entanglements which often span the nucleus several times. Alignment of these unpaired chromosome segments prior to synapsis was not observed although synaptonemal complex formation occurred at many sites along synapsing chromosomes. Exchange of pairing partners (multivalent formation), interlocking and pairing between non-homologous chromosomes were observed in the fully traced early zygotene nucleus. This confirms the observations by HoBOLTH (6) that these events are normal features of chromosome pairing in wheat. In fact, the above phenomena are not unexpected when one considers that wheat contains three sets of genetically and structurally related (homoeologous) chromosomes which have the potential to pair either with a homoeologous or a homologous partner. In addition, complex associations involving many chromosomes and bivalents (knots) were present in early to mid-zygotene nuclei and possibly arise in the following way. As synaptonemal complex formation proceeds at the onset of zygotene exchanges of pairing partners, simple hookings of bivalents or chromosomes as well as interlockings would tend eventually to concentrate the involved chromosome segments into knots. This is illustrated by the reconstruction in Figure 20. If synaptonemal 

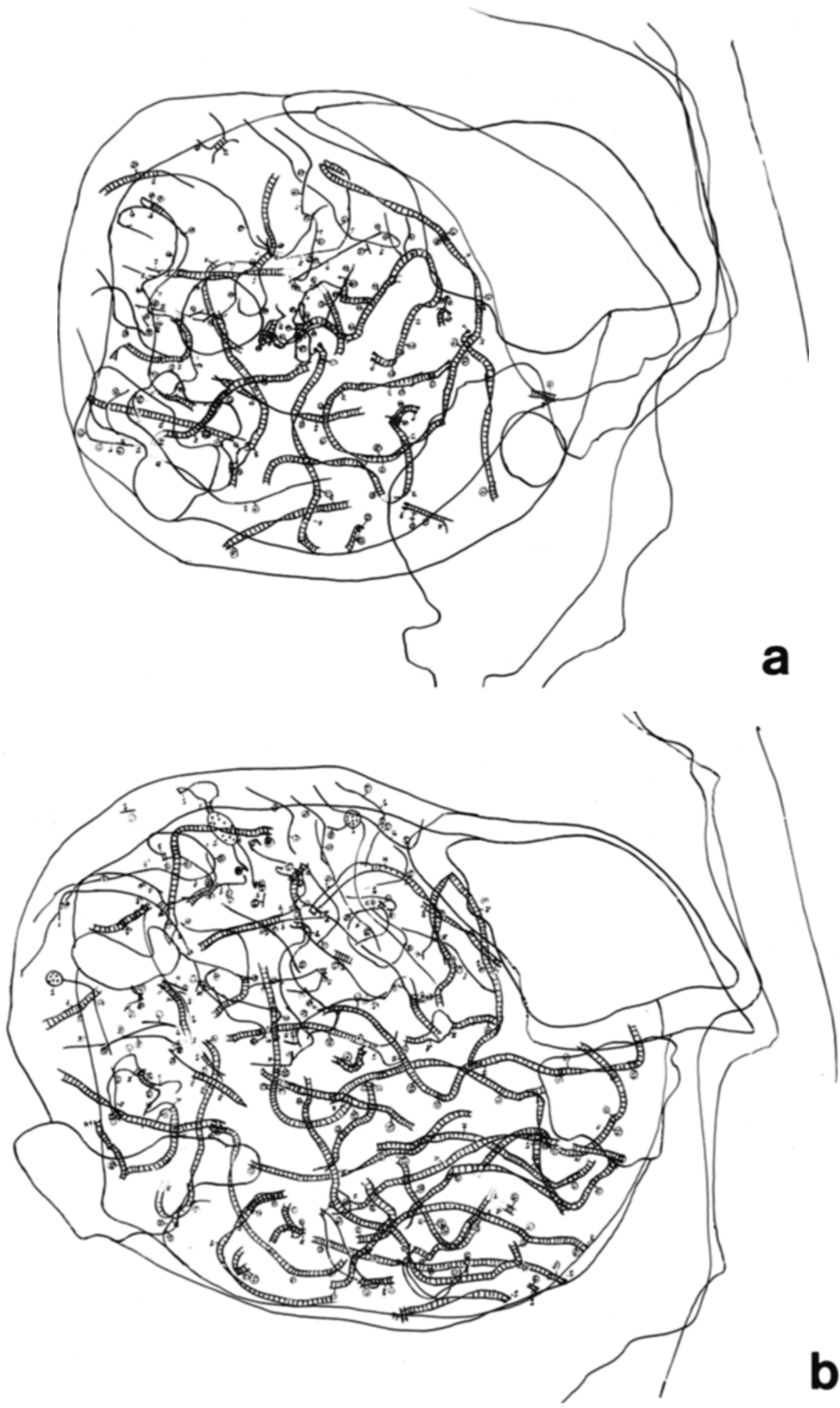

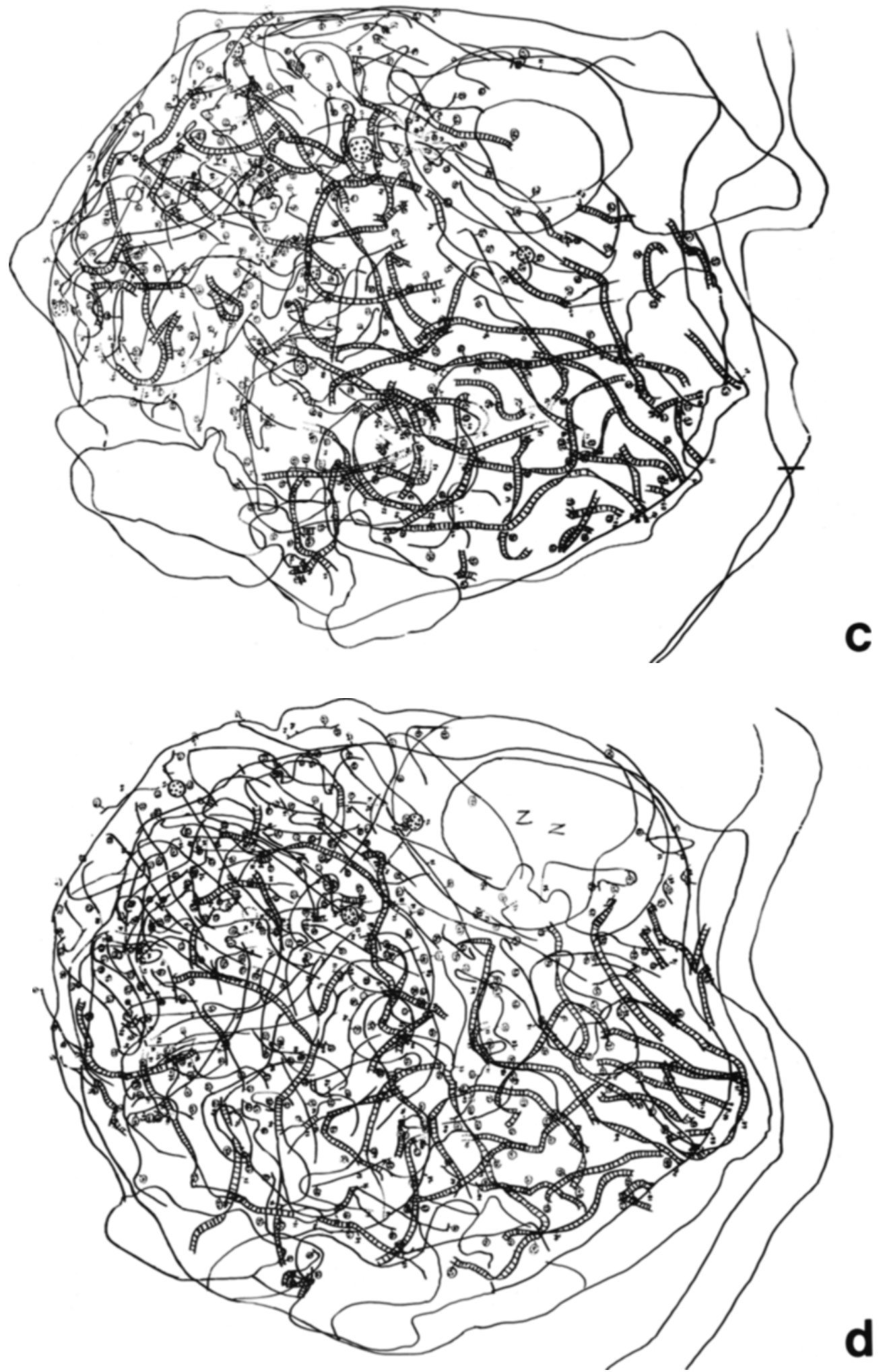

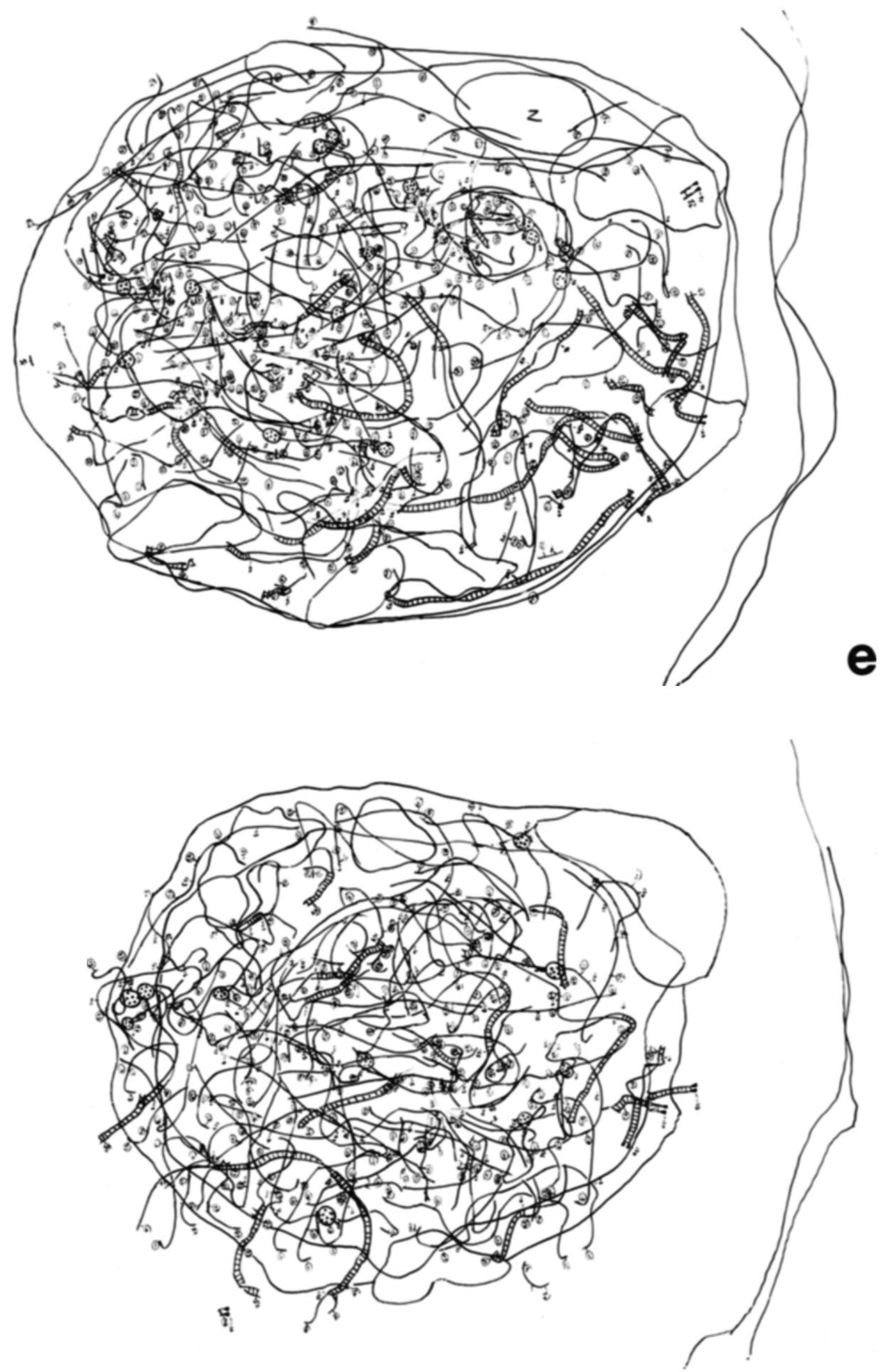

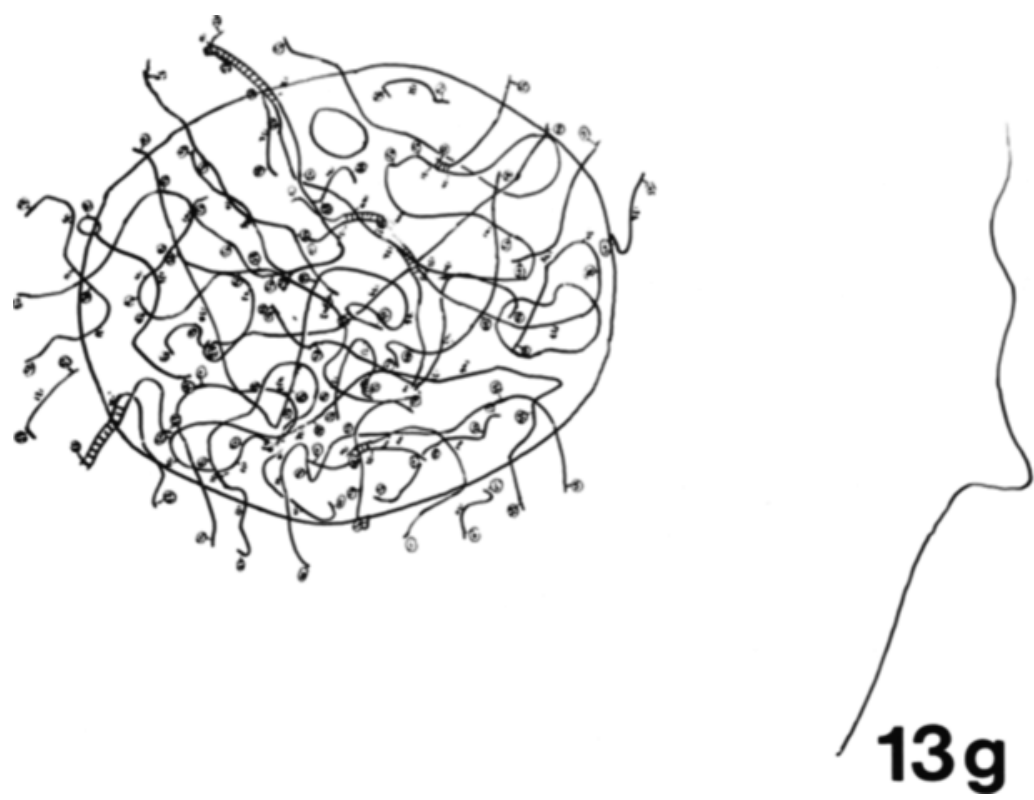

Figure 13. Photographic reductions of the original tracing sheets of the entire chromosome complement of an early zygotene nucleus. a. Sections 1-29, b. sections 30-49, c. sections 50-69, d. sections 70-89, e. sections 90-109, f. sections 110-129, g. sections 130-148. Unpaired lateral components are represented by single lines, synaptonemal complexes by parallel lines with cross hatching and membrane-bound telomeres by terminal thickenings of the lines. Note the polarisation of paired telomeres particularly in $b$-e and the uneven distribution of paired and unpaired chromosome segments throughout the nucleus. (Magnification approximately 7,200 times.) 

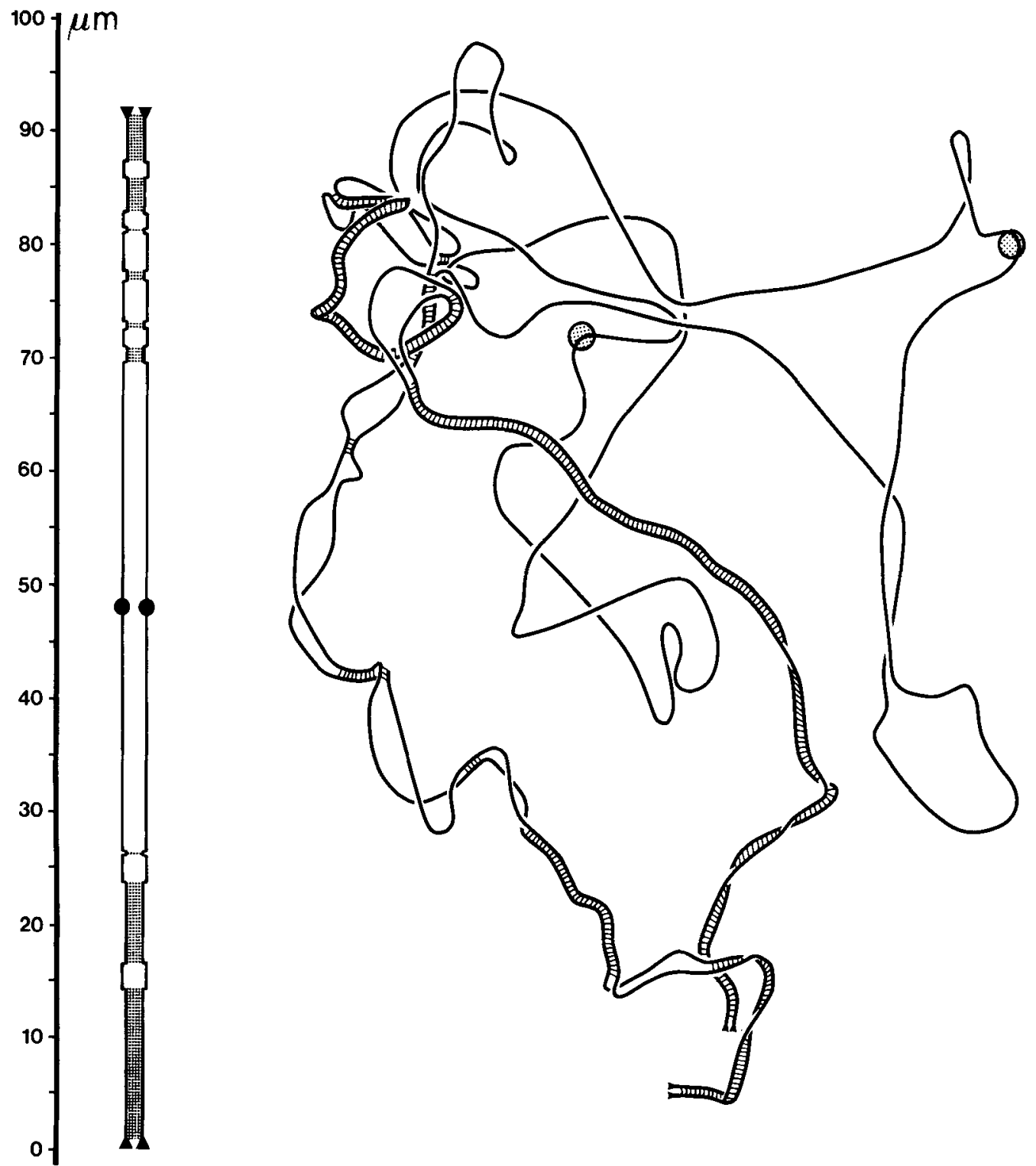

Figure 14. Reconstruction and diagrammatic representation of a bivalent at early zygotene. Unpaired lateral components are represented by single lines, synaptonemal complexes by parallel lines with cross hatching, centromeres by shaded circles and membrane-bound telomeres by terminal thickenings of the lines. Note the presence of multiple initiation sites of synaptonemal complex formation and the long unpaired middle region with widely spaced centromeres. 

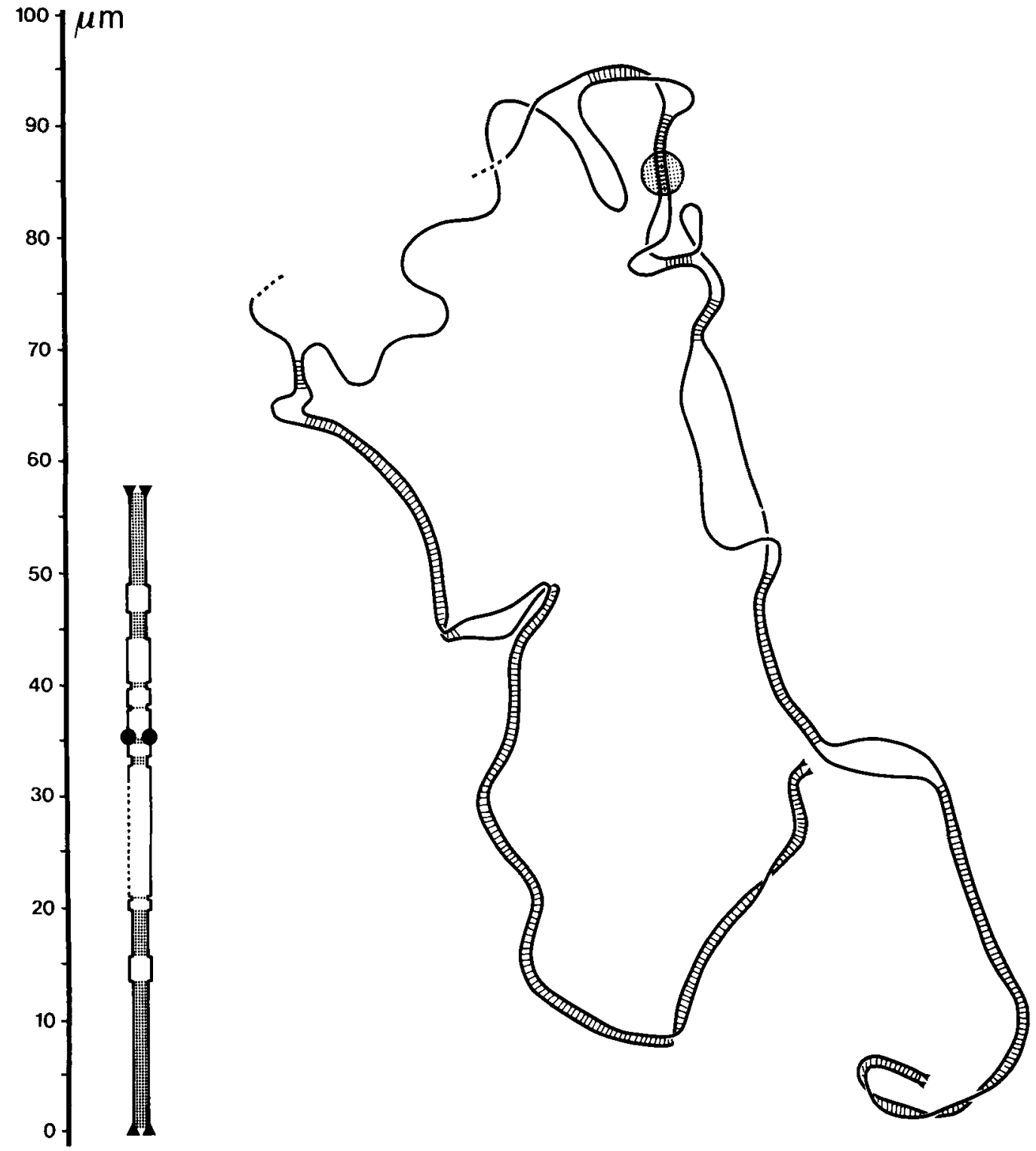

Figure 15. Reconstruction and diagrammatic representation of a bivalent at early zygotene. Sites of discontinuity of one lateral component is shown as a dotted line. (Other symbols as described in Figure 14). 

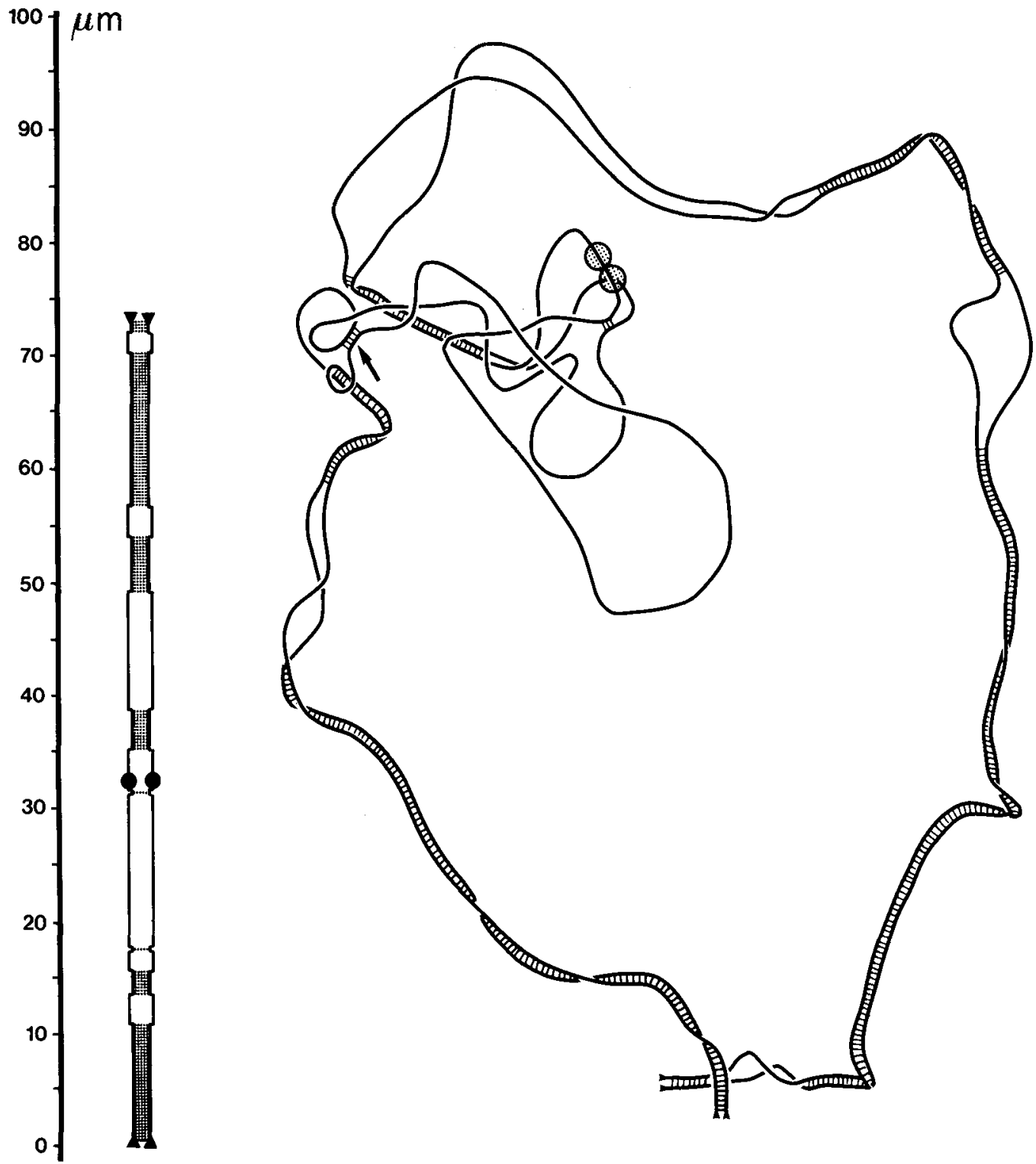

Figure 16. Reconstruction and diagrammatic representation of a bivalent at early zygotene. Note the inverted pairing indicated by an arrow. (Symbols as described in Figure 14). 


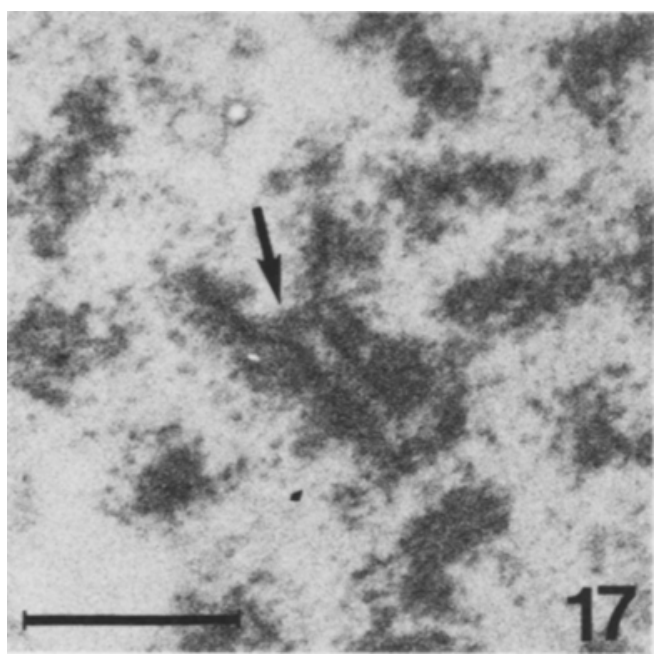

complex formation continued in the directions indicated by the arrows, a portion of the completed bivalent would eventually be present in the existing knot.

From mid-zygotene there is a gradual reduction in the frequency of knots. Simple hooking or entanglement could be resolved by some controlled movement of chromosomes relative to one another. But knots most likely also contain exchanges of pairing partners and interlockings, both of which would require a more sophisticated means of resolution. Bivalents or univalents that are entrapped between the lateral components of another bivalent could extricate themselves, but it is inconceivable how this could be achieved considering the length of the chromosomes. Where a chromosome loop of one bivalent interlocks that of another, resolution could proceed either by dissolution of the synaptonemal complex of one bivalent or by the breakage and reunion of one of the interlocked chromosome loops. Resolution by the latter method is well documented (e.g. 7,11) and several single lateral component breaks within largely paired bivalents have been observed in the early zygotene nucleus.

Multivalents can only be corrected by the dissolution and reassembly of the synaptonemal complex and have been shown to persist until late zygotene (6). The mechanism by which multivalents are transformed into bivalents is un-
Figure 17. Thin section through a PTA stained pairing fork (arrow). $(B a r=1 \mu \mathrm{m})$.

known but it is tempting to speculate that in mid-zygotene nuclei with the "reticulate" chromatin and in the later stages with highly condensed chromatin, some of the inter-chromosomal connections observed are manifestations of unresolved multivalents or those undergoing correction.

In conclusion, the present results confirm the presence of multivalents, and interlockings at zygotene as previously reported (6) and also show that extensive chromatin reorganization occurs throughout the zygotene stage. The precise nature of the control mechanism which ensures that only bivalents are present at pachytene is not understood, but it is known that the control resides in the long arm of chromosome $5 \mathrm{~B}$ (8, 14). Rasmussen and Holm (12) concluded that an analogous correction system was operational in autotetraploid Bombyx females due to the absence of crossing over which in males effectively prevented the transformation of multivalents. As crossing over occurs in wheat, HoBOLTH (6) forwarded the hypothesis that a gene product of chromosome $5 \mathrm{~B}$ somehow delays the time of crossing over until a stage when the pairing correction has been completed. Experimental tests of this hypothesis should be possible by investigating the time course of pairing correction in genotypes containing different doses of the $5 \mathrm{BL}$ arm. 


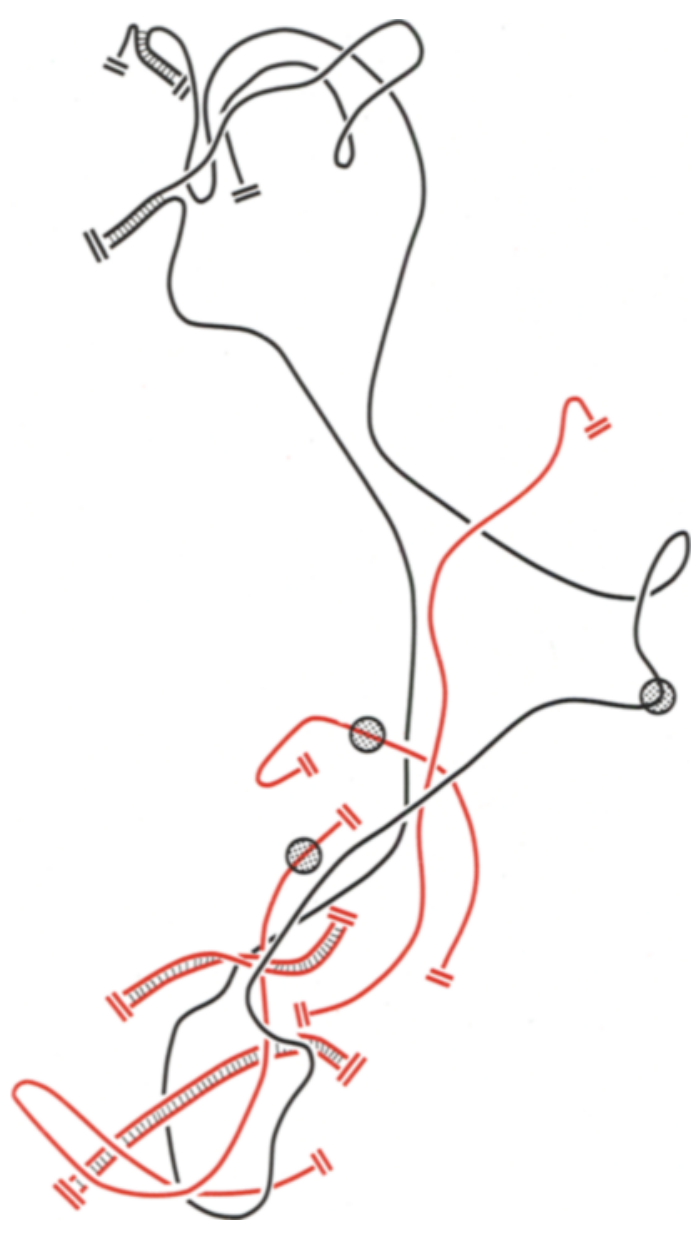

Figure 18. Detail from the reconstruction of the bivalent in Figure 14 showing some of the chromosome segments (red) which it interlocks. Chromosome segments are delimited by two perpendicular parallel lines. (Other symbols as described in Figure 14).

Figure 19. Reconstruction and diagrammatic representation of a pairing configuration involving six chromosomes at early zygotene. (Symbols as described in Figures $14,15$ and 18$)$. 

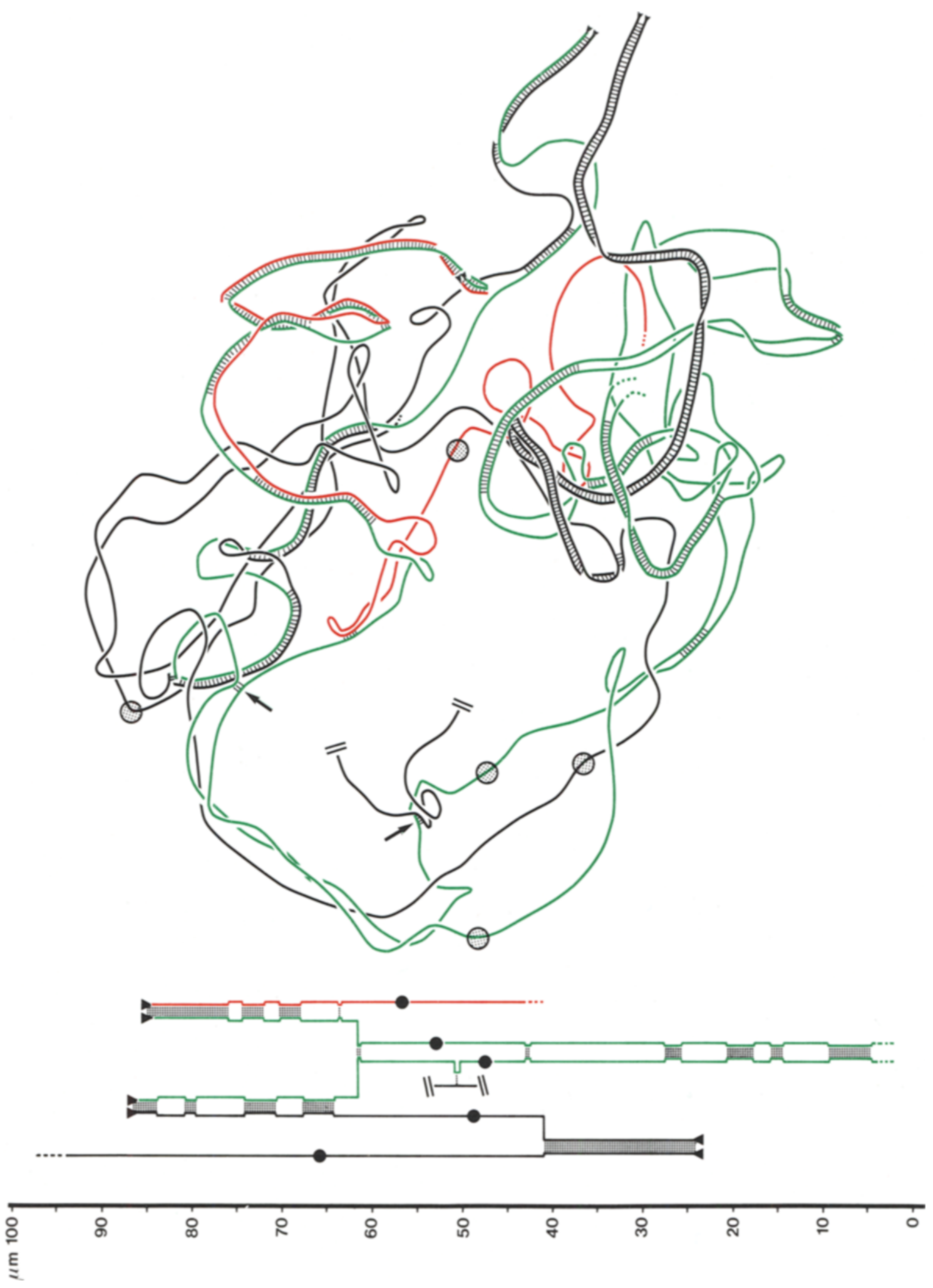


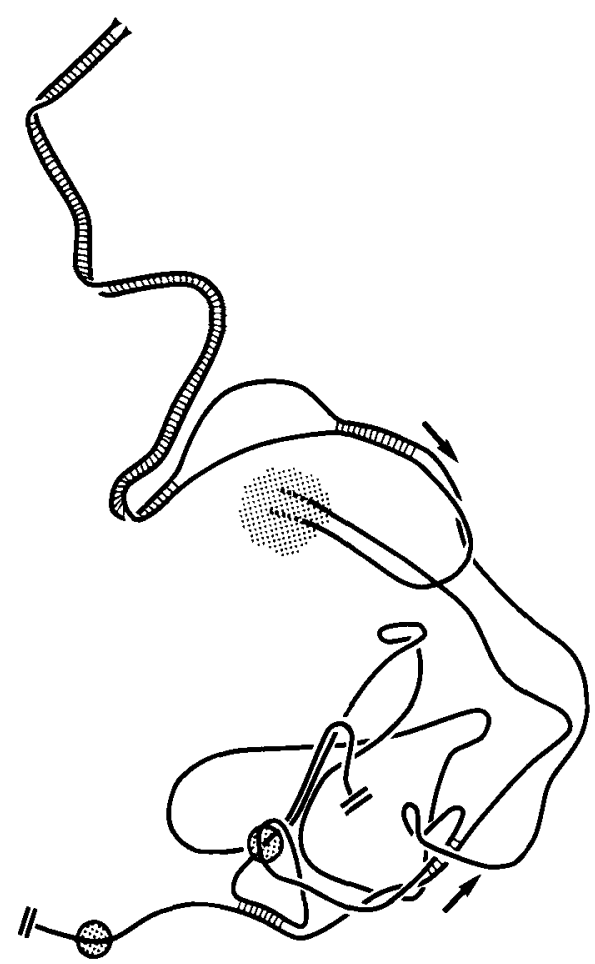

Figure 20. Reconstruction of a bivalent at early zygotene where one lateral component appears to be entrapped within a knot (hatched region). The second lateral component will be drawn into the knot when synaptonemal complex formation continues in the direction indicated by the arrows. (Other symbols as described in Figures 14, 15 and 18).

\section{ACKNOWLEDGEMENTS}

I wish to express my thanks to Professor DITER von Wettstein, Drs. Søren W. Rasmussen and Preben B. Holm for their continuous help, enthusiasm and encouragement during the course of this work. I am also indebted to Professor DITER von WetTSTEIN for extending to me the use of the excellent facilities of the Carlsberg Laboratory. I would further like to thank JEAN SAGE, JULia BOWler and Nina RaSMUSSEN for expert technical assistance and I acknowledge with gratitude the receipt of a one year fellowship award in the European Science Exchange Programme of the Royal Society of London. Financial support was provided through grant BIO-E417 DK(G) from the Commission of the European Communities to Professor D. voN WETTSTEIN.

\section{REFERENCES}

1. Avivi, L.A., M. Feldman \& M. Brown: An ordered arrangement of chromosomes in the somatic nucleus of common wheat, Triticum aestivum L. I. Spacial relatonships between chromosomes of the same genome. Chromosoma 86, 1-16 (1980)

2. Avivi, L.A., M. FELdMAN \& M. Brown: An ordered arrangement of chromosomes in the somatic nucleus of common wheat, Triticum aestivum L. II. Spacial relationships between chromosomes of different genomes. Chromosoma 86, 17-26 (1982)

3. Bennett, M., J.B. Smith, S. Simpson \& B. WELLS: Intranuclear fibrillar material in cereal pollen cells. Chromosoma 71, 289-332 (1979)

4. Feldman, M.: The effect of chromosomes 5B, 5D and 5A on chromosomal pairing in Triticum aestivum. Proc. Nat. Acad. Sci. U.S.A. 55, 1447- 1453 (1966)

5. GiLlies, C.B.: An ultrastructural analysis of chromosomal pairing in maize. Compt. Rend. Trav. Lab. Carlsberg 40, 135-161 (1975)

6. Hовоцтh, P.: Chromosome pairing in allohexaploid wheat var. Chinese Spring. Transformation of multivalents into bivalents, a mechanism for exclusive bivalent formation. Carlsberg Res. Commun. 46, 129-173 (1981)

7. HoLm, P.B.: Three dimensional reconstruction of chromosome pairing during the zygotene stage of meiosis in Lilium longiflorum (Thunb.). Carlsberg Res. Commun. 42, 103-151 (1977)

8. Окамото, M.: Asynaptic effect of chromosome V. Wheat Inf. Serv. 5, 6 (1957)

9. RASMUSSEN, S.W.: The meiotic prophase in Bombyx mori females analysed by three dimensional reconstructions of synaptonemal complexes. Chromosoma 54, 245-293 (1976)

10. Rasmussen, S.W.: Chromosome pairing in triploid females of Bombyx mori analysed by three dimensional reconstructions of synaptonemal complexes. Carlsberg Res. Commun. 42, 163-197 (1977)

11. Rasmussen, S.W. \& P.B. Holm: Human meiosis II. Chromosome pairing and recombination nodules in human spermatocytes. Carlsberg Res. Commun. 43, 275-327 (1978)

12. Rasmussen, S.W. \& P.B. Holm: Chromsome pairing in autotetraploid Bombyx females. Mechanism for exclusive bivalent formation. Carlsberg Res. Commun. 44, 101-125 (1979)

13. Rasmussen S.W. \& P.B. Holm: Mechanics of meiosis. Hereditas 93, 187-216 (1980)

14. Riley, R.\& V. Chapman: Genetic control of the 
cytologically diploid behaviour of hexaploid wheat. Nature, Lond. 182, 713-715 (1958)

15. Riley, R. \& C. Kempanna: The homoeologous nature of the non-homologous meiotic pairing in Triticum aestivum deficient for chromosome V (5B). Heredity 18, 287-306

16. SEARS, E.R.: Genetic control of chromosome pairing in wheat. Ann. Rev. Genet. 10, 31-51 (1976)
17. Sheridan, W.F. \& R.J. BarnetT: Cytochemical studies on chromosome ultrastructure. J. Ultrastruct. Res. 27, 216-229 (1969)

18. SPURR, A.R.: A low-viscosity epoxy resin embedding medium for electron microscopy. J. Ultrastruct. Res. 26, 31-43 (1969) 